\title{
Role of Cholinergic Anti-Inflammatory Pathway in Protecting Sepsis-Induced Acute Lung Injury through Regulation of the Conventional Dendritic Cells
}

\author{
Ruiting Li ${ }^{1},{ }^{1}$ Xuemei Hu, ${ }^{2}$ Huibin Chen, ${ }^{3}$ Yue Zhao, ${ }^{4}$ Xuehui Gao, ${ }^{1}$ Yin Yuan, ${ }^{1}$ \\ Huiling Guo, ${ }^{1}$ Haiyan Huang $₫{ }^{1},{ }^{1}$ Xiaojing Zou $₫{ }^{1},{ }^{1}$ Hong Qi $\odot{ }^{1},{ }^{1}$ Hong Liu $₫{ }^{1}{ }^{1}$ \\ and You Shang ${ }^{1}{ }^{1}$ \\ ${ }^{1}$ Department of Critical Care Medicine, Institute of Anesthesia and Critical Care Medicine, Union Hospital, Tongji Medical College, \\ Huazhong University of Science and Technology, Wuhan, Hubei 430022, China \\ ${ }^{2}$ Department of Nephrology, Taihe Hospital, Hubei University of Medicine, Shiyan, Hubei Province 442000, China \\ ${ }^{3}$ Department of Critical Care Medicine, Taihe Hospital, Hubei University of Medicine, Shiyan, Hubei Province 442000, China \\ ${ }^{4}$ Department of Critical Care Medicine, Jin Yin-tan Hospital, Wuhan, Hubei 430048, China
}

Correspondence should be addressed to Haiyan Huang; xhicuhhy@163.com, Xiaojing Zou; 2188628485@qq.com, Hong Qi; 1535796017@qq.com, Hong Liu; liuhongwuhan@126.com, and You Shang; you_shanghust@163.com

Haiyan Huang, Xiaojing Zou, Hong Qi, Hong Liu, and You Shang contributed equally to this work.

Received 2 October 2021; Revised 3 January 2022; Accepted 6 January 2022; Published 27 January 2022

Academic Editor: Marcella Reale

Copyright (c) 2022 Ruiting Li et al. This is an open access article distributed under the Creative Commons Attribution License, which permits unrestricted use, distribution, and reproduction in any medium, provided the original work is properly cited.

Background. The cholinergic anti-inflammatory pathway connects the immune response system and the nervous system via the vagus nerve. The key regulatory receptor is the $\alpha 7$-subtype of the nicotinic acetylcholine receptor $(\alpha 7 \mathrm{nAChR})$. Cholinergic anti-inflammatory pathway has been proved to be effective in suppressing the inflammation responses in acute lung injury (ALI). Dendritic cells (DCs), the important antigen-presenting cells, also express the $\alpha 7 \mathrm{nAChR}$. Past studies have indicated that reducing the quantity of mature conventional DCs and inhibiting the maturation of pulmonary DCs may prove effective for the treatment of ALI. However, the effects of cholinergic anti-inflammatory pathway on maturation, function, and quantity of DCs and conventional DCs in ALI remain unclear. Objective. It was hypothesized that cholinergic anti-inflammatory pathway may inhibit the inflammatory response of ALI by regulating maturation, phenotype, and quantity of DCs and conventional DCs. Methods. GTS-21 (GTS-21 dihydrochloride), an $\alpha 7 \mathrm{nAchR}$ agonist, was prophylactically administered in sepsis-induced ALI mouse model and LPS-primed bone marrow-derived dendritic cells. The effects of GTS-21 were observed with respect to maturation, phenotype, and quantity of DCs, conventional DCs, and conventional DCs2 (type 2 conventional DCs) and the release of DC-related proinflammatory cytokines in vivo and in vitro. Results. The results of the present study revealed that GTS-21 treatment decreased the maturation of DCs and the production of DC-related proinflammatory cytokines

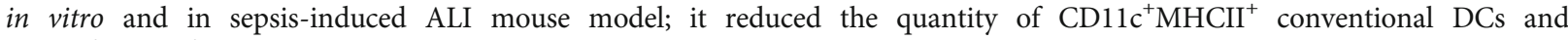

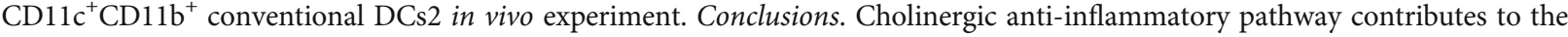
reduction in the inflammatory response in ALI by regulating maturation, phenotype, and quantity of DCs, conventional DCs, and conventional DCs2.

\section{Introduction}

Acute lung injury (ALI) and acute respiratory distress syndrome (ARDS) are common complications associated with both general and acute pulmonary diseases and are characterized by interstitial pulmonary edema, destruction of the alveolar-capillary barrier of the lungs, and excessive inflammatory response in the lung tissues [1]. Apart from the direct 
intrapulmonary factors, sepsis is the major extrapulmonary risk factor associated with ALI/ARDS. In more than half of the patients, sepsis progresses into ALI/ARDS, but there is not much clarity on the pathological mechanisms of sepsisinduced ALI/ARDS [2].

Both innate and adaptive immune response participate in the pathological process of ALI/ARDS [3, 4]. Innate immune response is the first line of defense when lung injury occurs and plays a key role in regulating its excessive inflammatory response. In addition, adaptive immune also plays an important role in inflammatory processes through regulating $\mathrm{T}$ cells, dendritic cells (DCs), and other immune regulatory cells $[3,4]$. DCs, T cells, and neutrophils in turn play key roles in the advancement of ALI through integrating innate and adaptive immunity, release of inflammatory cytokines, and activation of pro-inflammatory signaling pathway thereby controlling pulmonary inflammation [3, 4]. Hence, the inhibition of the pro-inflammatory pathway and the activation of the anti-inflammatory pathway of innate and adaptive immune response are crucial for the survival of patients $[3,4]$. The cholinergic anti-inflammatory pathway (CAP) establishes connection between the immune response and the nervous system through the vagus nerve. The key regulatory receptor is the $\alpha 7$-subtype of the nicotinic acetylcholine receptor ( $\alpha 7 \mathrm{nAChR})$, which is localized on the surface of immune cells. CAP has been proved to be effective in many pathologic processes including suppressing inflammation responses and improving endotoxemia, sepsis, cancer, and ischemia-reperfusion-induced ALI $[5,6]$. $\alpha 7 \mathrm{nAChR}$ signaling also have other biological effects, including antifibrosis, antitumor and anti-inflammation in chronic respiratory diseases $[6,7]$. Considering the significant effect of CAP on the pathological process of ALI, $\alpha 7 \mathrm{nAChR}$ has been considered as a potential intervention target for inhibiting excessive inflammatory response of ALI.

$\alpha 7 \mathrm{nAChR}$ is also distributed on the surface of cells of the immune system, in addition to that of the nervous system. Previous studies have reported that $\alpha 7 \mathrm{nAChR}$ is also expressed on the surface of DCs and plays a key role in the inhibition of immune response in DCs and T cells in the context of the CAP $[8,9]$. DCs, as important antigenpresenting cells (APCs), also serve as immune cells, playing the key role in immune response priming, and participate in the pathological process of ALI/ARDS [10-12].

Previous studies have indicated that reducing the quantity of mature conventional DCs (cDCs) and inhibiting the maturation of pulmonary DCs may prove effective for the treatment of ALI $[13,14]$. CD $11 c^{+}$DCs were found to be the key protecting cells in transfusion-related acute lung injury (TRALI), and this protection occurs via IL-10. Deple-

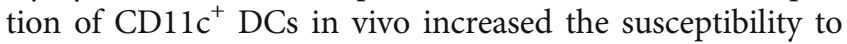
induce TRALI $[14,15]$. However, the effects of CAP on the maturation and function of DCs and cDCs in ALI/ARDS remain unclear.

Therefore, it was hypothesized that the activation of CAP may inhibit the excessive inflammatory response of ALI by mediating maturation, phenotype, and quantity of DCs and cDCs. This may have been proved to be a new intervention in the treatment of ALI.

\section{Material and Methods}

2.1. Animals. SPF C57BL/J6 male mice (aged 6-8 weeks, weight $20 \pm 2 \mathrm{~g}$ ) were purchased from the Experimental Animal Centre of Hubei province (Wuhan, China). All experimental procedures were performed in compliance with the National Institutes of Health Guidelines and were approved by Huazhong University of Science and Technology (Wuhan, China) experimental animal ethics committee.

2.2. Establishment of ALI Mouse Model and Administration of GTS-21. All experimental mice were randomly divided into 4 groups ( $n=4-6$ mice per group): control group; sepsis-induced ALI group; GTS-21 (GTS-21 dihydrochloride, a $\alpha 7 \mathrm{nAchR}$ agonist; Abcam, Cambridge, UK) positive control group; and ALI-treated by GTS-21 group. No differences in the food intake or body weight were observed among the groups. In order to induce ALI, the mice in the ALI group and ALI treated by GTS-21 group were administered with the intraperitoneal (i.p.) injection of $200 \mu \mathrm{L}$ sterile phosphate-buffered saline (PBS), which contained $15 \mathrm{mg} / \mathrm{kg}$ lipopolysaccharide (LPS, Sigma Aldrich, St. Louis, MO, USA). The control and GTS-21-positive control groups were administered with only $200 \mu \mathrm{L}$ of sterile PBS at the same time points. In order to activate the CAP, the positive control and treatment groups prophylactically received an i.p. injection of $4 \mathrm{mg} / \mathrm{kg}$ GTS-21, diluted in $200 \mu \mathrm{L}$ of PBS, $30 \mathrm{~min}$ before the administration of LPS [13]. All the mice were sacrificed after blood and the broncho-alveolar lavage fluid (BALF) were collected, $24 \mathrm{~h}$ after the LPS injection. This was followed by the removal of the lungs and spleens, which were temporarily stored in RPMI-1640 supplemented with $10 \%$ fetal bovine serum (FBS) (HyClone; Logan, UT, USA) at $4^{\circ} \mathrm{C}$ for further investigation.

2.3. Collection of BALF. The lungs were flushed three times with $0.5 \mathrm{~mL}$ PBS via a tracheal cannula to collect BALF. The BALF was centrifuged, then the supernatant was collected for the subsequent analysis of cytokine levels [13].

2.4. Preparation of Bone Marrow-Derived DCs (BMDCs). The bone marrow was extracted from the femur bones of C57BL/J6 mice (age: 5-8 weeks). On day 0, bone marrow mononuclear cells (MNCs) were seeded at $1-2 \times 10^{6}$ cells/ $\mathrm{mL}$ in $15 \mathrm{~mL}$ RPMI-1640 that contained $10 \mathrm{ng} / \mathrm{mL}$ granulocyte-macrophage colony-stimulating factor (GMCSF) (PeproTech, Rocky Hill, NJ, USA) and $10 \mathrm{ng} / \mathrm{mL}$ recombinant mouse IL-4 (rmIL-4) (PeproTech, Rocky Hill, NJ, USA), then they were cultured in an incubator under the following conditions: $37^{\circ} \mathrm{C}$ and $5 \% \mathrm{CO}_{2}$. On days 3 and 5 , $10 \mathrm{~mL}$ freshly prepared RPMI-1640 containing $10 \mathrm{ng} / \mathrm{mL}$ rmIL-4 and GM-CSF were added to the plates to change the culture medium. On day 7, the BMDCs were collected and resuspended in RPMI-1640 medium at a density of $1-2 \times$ $10^{6}$ cells $/ \mathrm{mL}$; the purity of the immature DCs in in the samples was at least $90 \%$. The BMDCs were then incubated with or without LPS $(100 \mathrm{ng} / \mathrm{mL})$ and GTS-21 (100 mM) for $24 \mathrm{~h}$ [13]. The BMDC culture supernatants were collected for subsequent enzyme-linked immunosorbent assay (ELISA) assay. The BMDCs were frozen at $-80^{\circ} \mathrm{C}$ for further analysis. 
2.5. ELISA. The levels of cytokines [interleukin-6 (IL-6), tumor necrosis factor-alpha (TNF- $\alpha$ ), IL-12p40, IL-18, IL$1 \beta$, and high mobility group box 1 (HMGB1)], secreted by DCs, were measured by ELISA, according to manufacturer's instructions. The ELISA detection kits of IL- 6 , TNF- $\alpha$, IL$12 \mathrm{p} 40$, IL-18, and IL-1 $\beta$ were purchased from eBioscience (San Diego, CA, USA), and the HMGB1 ELISA kit was purchased from LifeSpan BioSciences, Inc. (Seattle, Wash).

2.6. Flow Cytometric Analysis. Lung single-cell suspensions and BMDC suspensions collected from different groups were stained with PE anti-mouse CD11c (eBioscience, San Diego, CA, USA) and APC-Cy7 anti-mouse F4/80 (Biolegend Inc., San Diego, CA, USA). DCs were marked as $\mathrm{CD} 11 \mathrm{c}^{+}$and $\mathrm{F} 4 / 80^{-}$cells. This was followed by analysis of the phenotype and the maturation of DCs using FITC anti-mouse CD80, FITC anti-mouse major histocompatibility complex II (MHCII), FITC anti-mouse CD40 (eBioscience, San Diego, CA, USA), and APC anti-mouse CD86 (Biolegend Inc., San Diego, CA, USA) stains. Among them, cDCs were marked as $\mathrm{CD}_{11 \mathrm{c}^{+} \mathrm{MHCII}}{ }^{+}$doublepositive cells. In addition, the cDCs2 (type $2 \mathrm{cDCs}$ ) in lung and spleen MNCs were stained as $\mathrm{CD} 11 \mathrm{c}^{+} \mathrm{CD} 11 \mathrm{~b}^{+}$doublepositive cells using PE anti-mouse CD11c and APC antimouse CD11b (Biolegend Inc., San Diego, CA, USA). All the cells, after being washed with PBS, were analyzed using Flow Cytometer (FACSAria ${ }^{\mathrm{TM}}$ III, BD Biosciences, USA). The FlowJo software (FlowJo LLC, Ashland, Ore) was used to analyze the data.

2.7. Statistical Analysis. All results are presented as mean \pm standard deviation (SD). All experiments were repeated more than thrice ( $n=4-6$ mice/group). Presented data are one representative experiment of three repeats. Statistical analysis was performed using the one-way ANOVA. Data were analyzed using the SPSS 22.0 software (IBM SPSS, Chicago, IL, USA). A $p$ value of $<0.05$ was considered significant.

\section{Results}

3.1. CAP Inhibited the Maturation of DCs in Sepsis-Induced ALI Mouse Model. To assess the role of CAP on differentiation and maturation of DCs in sepsis-induced ALI mouse model (i.p. injected LPS), GTS-21, the $\alpha 7 \mathrm{nAchR}$ agonist, was i.p. injected $30 \mathrm{~min}$ before LPS intervention in order to activate the CAP. After $24 \mathrm{~h}$ of the administration of LPS, suspensions of lung MNCs were prepared. Flow Cytometry was used to analyze the expression of antigen-presenting molecule MHCII and costimulatory molecules (CD80, CD86, and CD40) on surface of $\mathrm{CD} 11 \mathrm{c}^{+} \mathrm{F} 4 / 80^{-}$DCs (Figure 1(a)). Mature differentiation of DCs was characterized by the expressions of CD80, CD86, CD40, and MHCII on their surface [16]. Lung MNCs were first stained with $\mathrm{PE}$ anti-mouse CD11c and APC-Cy7 anti-mouse F4/80, and the DCs were detected as $\mathrm{CD} 11 \mathrm{c}^{+} \mathrm{F} 4 / 80^{-} \mathrm{MNCs}$ (Figure 1(a)). It was found that the percentages of MHCII, CD40, CD80, and CD86 on surface of DCs obviously increased in the sepsis-induced ALI group when compared to the control and GTS-21-positive control mice group, and the increase in MHCII, CD40, and CD86 was reduced through GTS-21 intervention (Figures 1(b) and 1(c)). Therefore, CAP has the potential to inhibit mature differentiation of DCs in sepsis-induced ALI mouse model.

3.2. Downregulation of the Levels of DC-Related Proinflammatory Cytokines in ALI Mouse Model through GTS21 Treatment. To examine the effect of CAP on DC-related pro-inflammatory cytokines, the levels of IL-6, TNF- $\alpha$, IL-18, and IL- $1 \beta$ in the serum and BALF of different group mice were detected. The release of TNF- $\alpha$, IL-6, IL-18, and IL- $1 \beta$ in the serum along with BALF was observed to be significantly higher in the ALI groups when compared to that in the control and GTS-21-positive control mice group. On the other hand, the administration of GTS-21 caused a decrease in the level of cytokines TNF- $\alpha$, IL- 6 , and IL-18 in the serum and the release of cytokines TNF- $\alpha$ and IL- $1 \beta$ in BALF of ALI mice (Figures 2(a)-2(d)). In the previous studies conducted by the investigators, it was found that GTS-21 was involved in the downregulation of the expression and the release of two other DC-related cytokines (IL-12p40 and HMGB1) in ALI [13]. Taking into consideration the findings of the current research and that of the previously conducted study, it can be inferred that CAP is responsible for a significant decrease in the levels of these pro-inflammatory cytokines released by DCs in vivo.

3.3. CAP Reduced the Quantity of $c D C s$ and $c D C s 2$ in ALI Mouse Model. The DCs have two subsets: plasmacytoid DCs (pDCs) and cDCs. cDCs, the predominating DC population, are preferentially localized in the lung interstitium and play a significant role in systematic inflammatory response [17]. $\mathrm{cDCs}\left(\mathrm{CD} 11 \mathrm{c}^{+} \mathrm{MHCII}^{+}\right)$in the lung are represented by $\mathrm{CD} 11 \mathrm{c}^{+} \mathrm{CD} 103^{+} \mathrm{DCs}$ (type $1 \mathrm{cDCs}, \mathrm{cDCs} 1$ ) and $\mathrm{CD}_{11} \mathrm{c}^{+} \mathrm{CD} 11 \mathrm{~b}^{+} \mathrm{DCs}(\mathrm{cDCs} 2)$, and $\mathrm{cDCs} 2$ in the lungs were identified as $\mathrm{CD} 11 \mathrm{~b}^{+}$. In the spleen, $\mathrm{cDCs}$ are also classified into the subsets according to the CD11b marker expression [17-19]. It was recently reported that the population of $\mathrm{CD}_{11} \mathrm{c}^{+} \mathrm{CD} 11 \mathrm{~b}^{+} \mathrm{DCs}(\mathrm{cDCs} 2)$ sense pathogens, produce proinflammatory cytokines, and drive naive $\mathrm{CD}^{+} \mathrm{T}$ cells toward distinct effector subsets [20]. cDCs were marked as

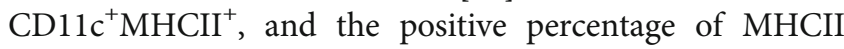
not only represented the maturation of DCs but also represented the total population of $\mathrm{cDCs}$. As depicted in the part of MHCII in Figures 1(b) and 1(c), the percentage of cDCs in the lung tissues of the ALI group showed a significant increase when compared to those from the control mice, and GTS-21 treatment caused a significant downregulation of the percentage of cDCs compared with that in the ALI mice. In order to examine the role of GTS- 21 on cDCs2, the $\mathrm{CD} 11 \mathrm{c}^{+} \mathrm{CD} 11 \mathrm{~b}^{+}$double-positive DCs were stained and separated from lung and spleen MNCs of different groups. The percentages of cDCs 2 in the lung and spleen were markedly higher in the ALI group as compared to that in the control and GTS-21-positive control group. GTS-21 administration downregulated the percentages of pulmonary and splenic cDCs2 when compared with that in the ALI group (Figures 3(a)-3(c)). These findings suggested that CAP activation can result in the downregulation of the number of $\mathrm{cDCs}$ and $\mathrm{cDCs} 2$ in ALI. 

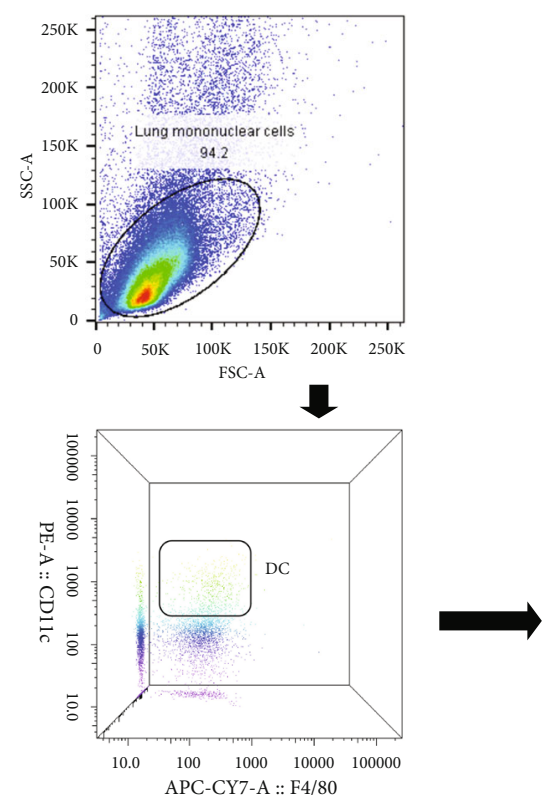

(a)

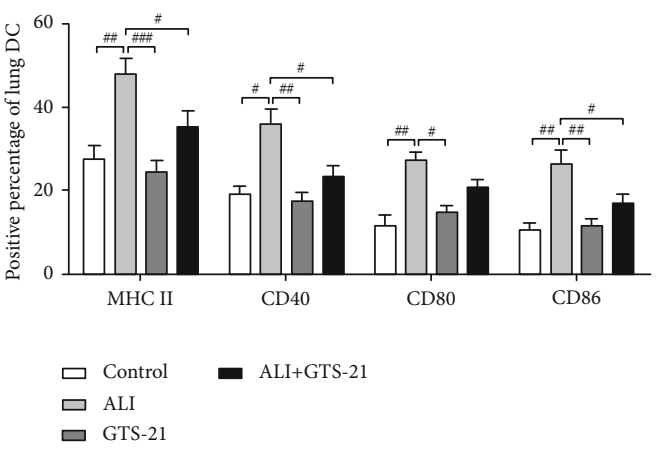

(c)
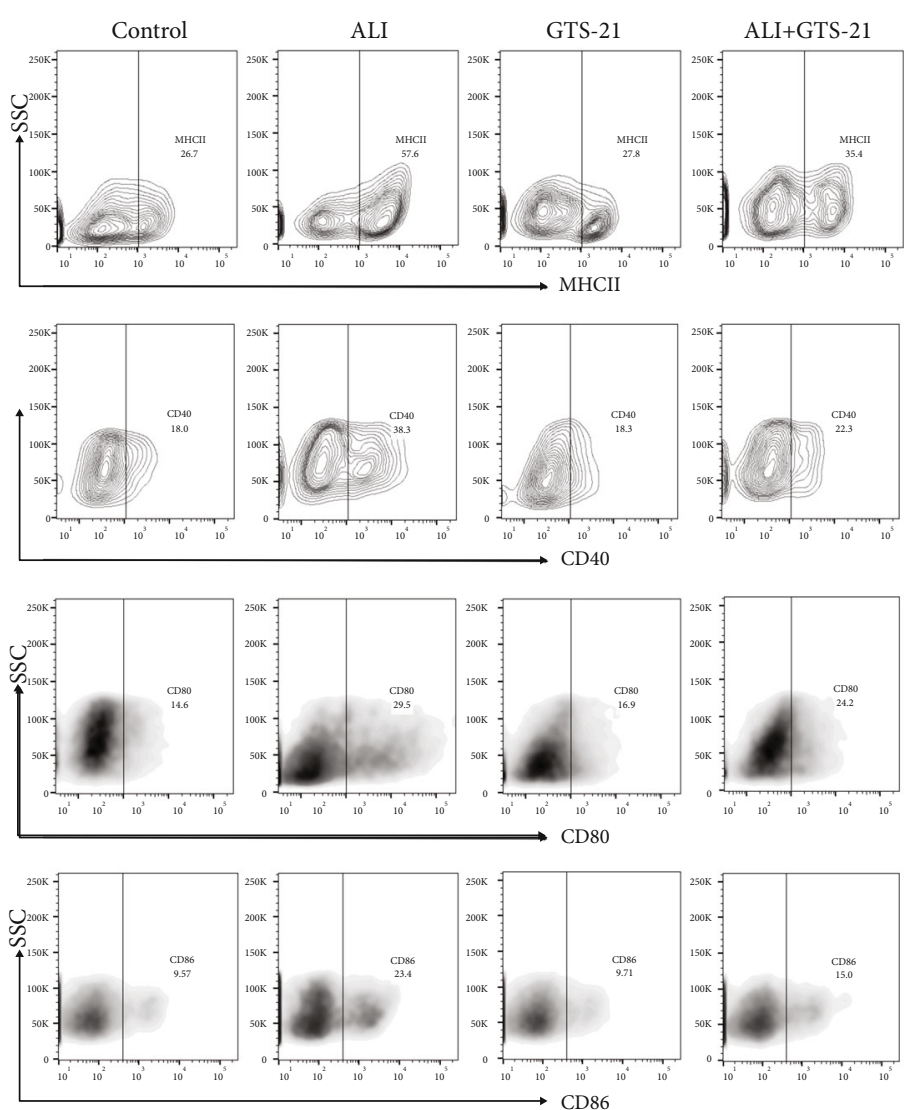

(b)

FIGURE 1: GTS-21 administration inhibited the maturation of DCs in the lungs of ALI induced by sepsis. (a) Flow cytometry analysis was used to examine the CD11 $\mathrm{c}^{+} \mathrm{F} 4 / 80^{-}$lung DCs isolated from lung MNCs of mice. (b, c) Detecting the percentages of MHCII-, CD40-, CD80-, and CD86-positive expression on the surface of lung DCs. $n=4-6$ mice/group. All data are shown as mean \pm SD. ${ }^{\#} p<0.05,{ }^{\# \#} p<0.01$, ${ }^{\# \# \#} p<0.001$.

3.4. GTS-21 Inhibited the Maturation of DCs In Vitro. To analyze the effect of CAP on mature differentiation of DCs in vitro, BMDCs were stimulated with LPS to induce maturation, and GTS-21 was added to observe whether it inhibited the maturation process of BMDCs. Figure 4(a) depicts the morphology of BMDCs (numerous extended dendrites in different directions from the cell body). Flow Cytometry was used to analyze the expression of MHCII and CD80 on the surface of DCs. It was found that the percentages of MHCII and CD80-positive expression were markedly elevated in the case of LPS-primed BMDCs group in comparison to that in the control BMDCs group. On the other hand, GTS-21 significantly inhibited the expression of MHCII on LPS-primed BMDCs (Figures 4(b) and 4(c)). The obtained data suggested that CAP inhibited the differentiation of DCs into mature cells in vitro.

3.5. GTS-21 Downregulated the Levels of DC-Related Proinflammatory Cytokines In Vitro. ELISA analysis was per- formed to analyze the effect of GTS-21 on the release of DC-related pro-inflammatory cytokines from LPS-primed BMDCs for measuring the levels of IL-6, TNF- $\alpha$, IL-18, IL$1 \beta$,IL-12p40, and HMGB1 in the BMDC culture supernatant. It was observed that the production of IL-6, TNF- $\alpha$, IL-18, IL-1 $\beta$, IL-12p40, and HMGB1 was markedly upregulated due to LPS stimulation when compared to that in the control group. On the other hand, GTS-21 treatment markedly decreased the production of the IL- 6 , TNF- $\alpha$, IL-18, IL$1 \beta$, IL-12p40, and HMGB1 cytokines in the BMDC culture supernatant (Figures 5(a)-5(f)). These results suggested that CAP has the potential to inhibit the production of proinflammatory cytokines from DCs in vitro.

\section{Discussion}

ALI is characterized by inflammatory cell infiltration and recruitment to the injured lung interstitium. Among them, DCs are the APCs that play a significant role in 


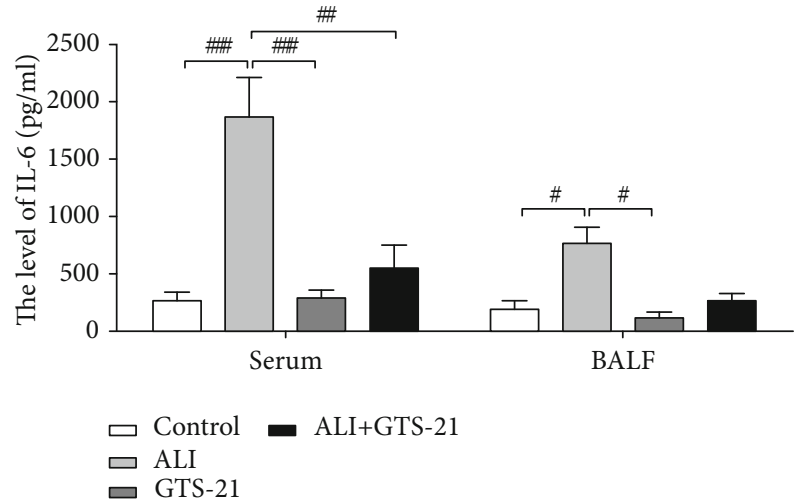

(a)

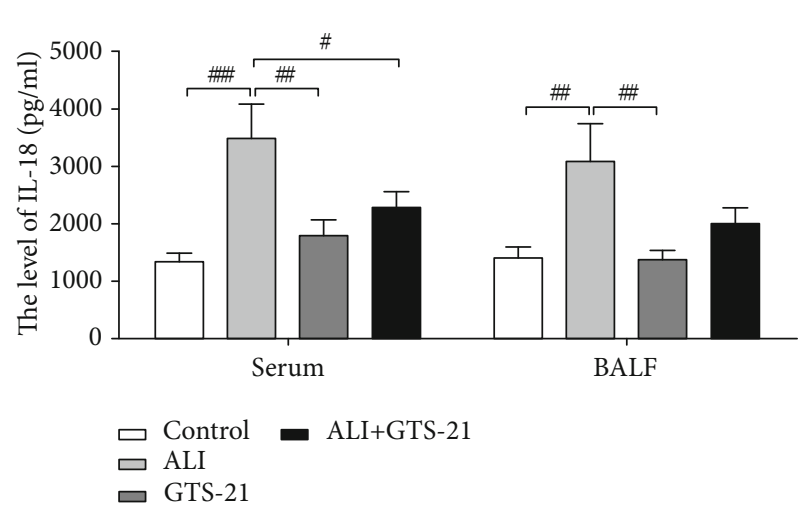

(c)

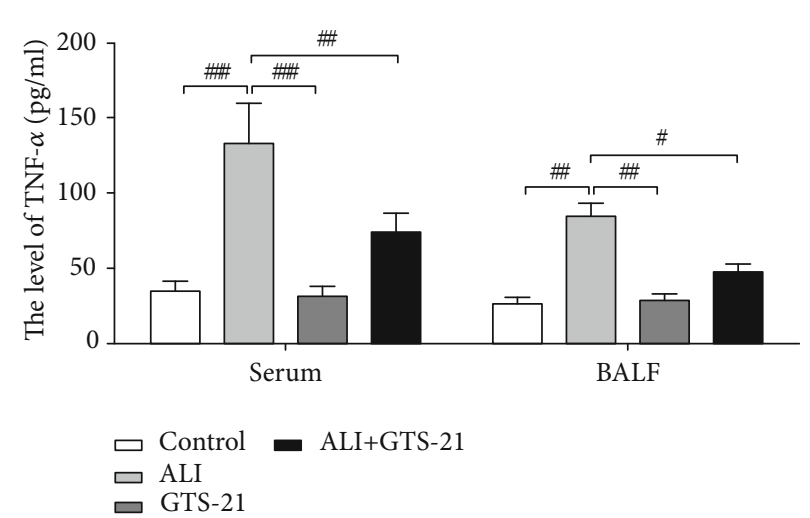

(b)

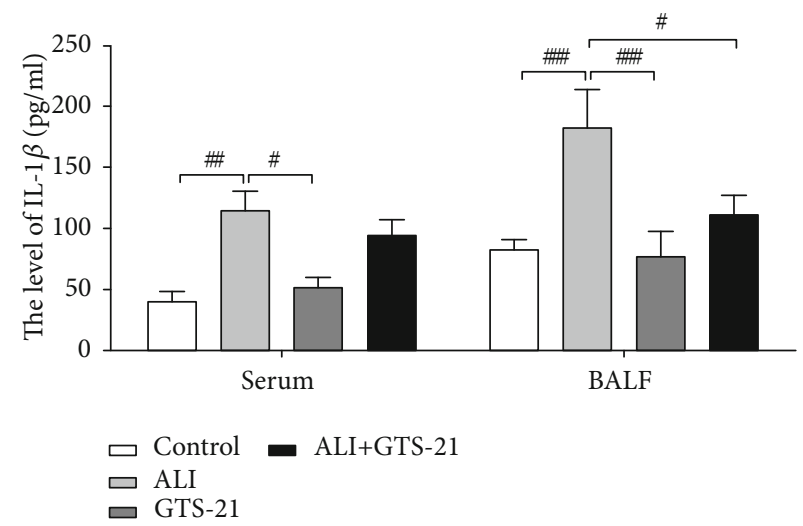

(d)

FIGURE 2: GTS-21 administration reduced the releases of DC-related proinflammatory cytokines in the serum and BALF of sepsis-induced ALI mice. (a-d) The productions of cytokines IL-6, TNF- $\alpha$, IL-18, and IL- $1 \beta$ in the serum and BALF were measured by ELISA. $n=4-6$ mice/group. All data are shown as mean \pm SD. ${ }^{\#} p<0.05,{ }^{\# \#} p<0.01,{ }^{\# \#} p<0.001$.

inflammatory response in the case of sepsis-induced lung injury. Numerous studies have suggested that by adopting different ways to decrease recruitment of activated DCs and $\mathrm{cDCs}$ in the lung may reduce the incidences of lung injury $[11,12]$. In our present study, it was found that GTS-21 treatment decreased the maturation of DCs and the release of DC-related pro-inflammatory cytokines in vitro and in sepsis-induced ALI mouse model and reduced the quantity of $\mathrm{CD} 11 \mathrm{c}^{+} \mathrm{MHCII}^{+} \mathrm{cDCs}$ and $\mathrm{CD} 11 \mathrm{c}^{+-}$

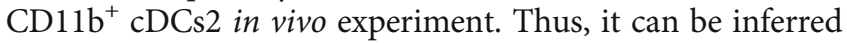
that the activation of CAP may cause decreased inflammatory response in ALI through the regulation of maturation, phenotype, and quantity of DCs, cDCs, and cDCs2.

The acute inflammatory response in ALI not only includes the activation of innate and adaptive immune but also certain specific activities of the autonomic nervous system. The vagus nerve is considered to perform antiinflammatory functions, by inhibiting the release of proinflammatory cytokines and protecting the lung from pathological injury; this anti-inflammatory function of the vagus nerve is termed as CAP [21]. It has been confirmed that CAP protects the lungs from infection and sepsis. In this signaling pathway, $\alpha 7 \mathrm{nAChR}$ is a key target receptor that can attenuate the production of pro-inflammatory mediators from inflammatory cells, such as macrophages, DCs and neutro- phils, at the same time as expressing itself on the surface of these inflammatory cells $[9,22]$. Recent studies suggested that $\alpha 7 \mathrm{nAChR}$ inhibited the activation of downstream inflammasomes, decreased the secretion of proinflammatory cytokines and chemokines from macrophages, which play an important role in regulating the progression of lung injury, and reduced lung inflammatory injury [23, 24]. $\alpha 7 \mathrm{nAchR}$ also can reduce the expression of Caspase- 1 and IL- $1 \beta$ associated with pyroptosis, a programmed cell death pathway which plays an important role in the process of lung injury [23]. A number of studies have shown that the activation of CAP inhibited differentiation and maturation of DCs, reduced DC-associated cytokine release, and ameliorated the inflammatory responses in the body [9]. However, the relationship between CAP and DCs in ALI remains unclear. In studies conducted by the investigators, it was found that the anti-inflammatory effect of the CAP agonist GTS-21 on DCs in sepsis-induced ALI: GTS-21 significantly inhibited the expression of MHCII, CD40, and CD86 on the surface of DCs and also the release of DC-related proinflammatory cytokines (IL-6, TNF- $\alpha$, IL-18, IL- $1 \beta$, IL12p40, and HMGB1).

DCs through identification of the antigens of the pathogens present antigens to activate $\mathrm{T}$ cells and initiate antigenspecific cellular immune responses, which are associated 

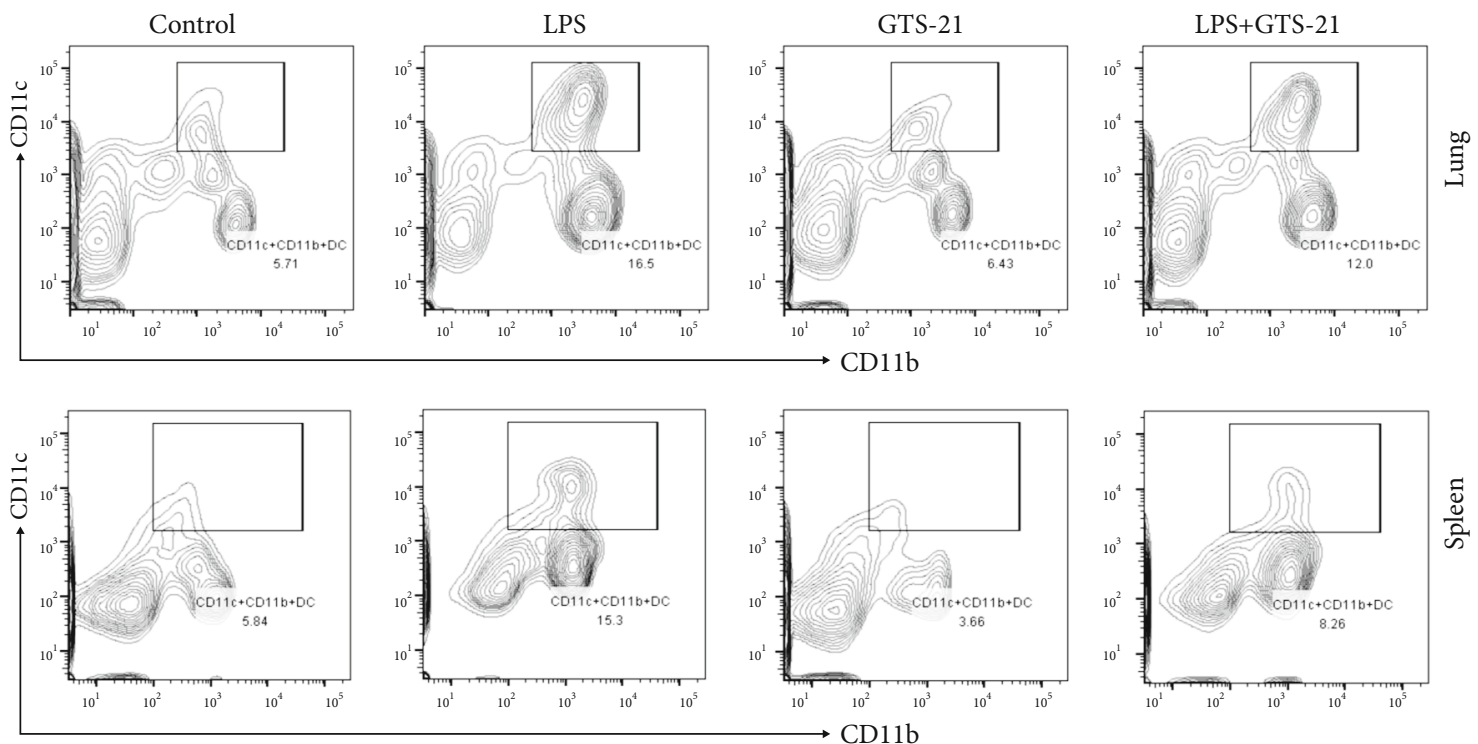

(a)

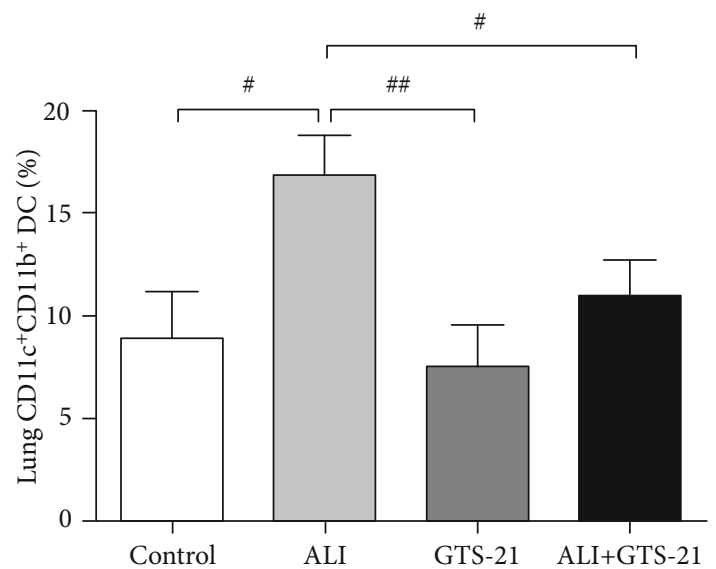

(b)

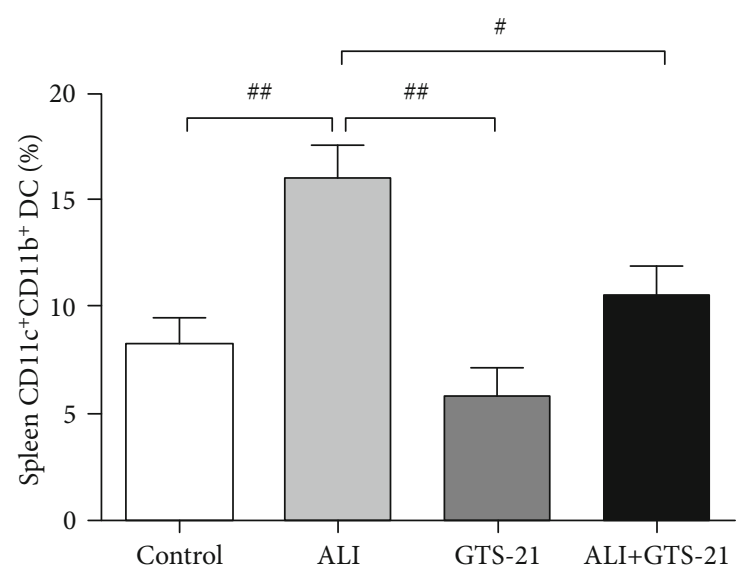

(c)

FIGURE 3: GTS-21 treatment reduced the number of cDCs2 in the lungs and spleens of sepsis-induced ALI mice. (a) Flow cytometry analysis was used to examine the CD11 $\mathrm{c}^{+} \mathrm{CD} 11 \mathrm{~b}^{+} \mathrm{cDCs} 2$ isolated from the lung and spleen MNCs of mice. $(\mathrm{b}, \mathrm{c})$ The relative percentages of lung and spleen $\mathrm{CD} 11 \mathrm{c}^{+} \mathrm{CD} 11 \mathrm{~b}^{+}$cells in different groups. $n=4-6$ mice/group. All data are shown as mean $\pm \mathrm{SD}$. ${ }^{\#} p<0.05,{ }^{\# \#} p<0.01,{ }^{\# \# \#} p<0.001$.

with many diseases, and play a critical role in the pathological process of ALI. In mouse and human beings, there are different types of DCs with different phenotypes, functions, and localizations that form an immune system, which is distributed across all organs of the body and is responsible for immune-surveillance $[19,25,26]$. DCs can be classified into two main lineages, $\mathrm{cDCs}$ and $\mathrm{pDCs}$, which are crucial for the priming phase of the immune response. A number of studies have reported that marked increase in mature $\mathrm{CDCs}$ plays a key pro-inflammatory role in lung injury. As ALI progresses, cDCs express MHCII and CD80, which are rapidly accumulated in the lung interstitium, resulting in the production of a range of inflammatory mediators $[11,12,26,27]$. These findings are consistent with the findings of the current study. Moreover, the data obtained in the current study also confirmed immunomodulatory effects of CAP on cDCs in pathological process of ALI using GTS-21. GTS-21 caused significant reduction in the quantity of $\mathrm{CD} 1 \mathrm{c}^{+} \mathrm{MHCII}^{+}$
cDCs in LPS-induced mice model and in in vitro experiment. The data suggested that the anti-inflammatory activity of the CAP in ALI can be attributed to regulation of the number and accumulation of cDCs.

cDCs can also be further divided into two main subsets: cDCs 1 and cDCs2. In mouse, the cDCs1 are characterized by the expression of CD103 and cDCs2 phenotype being $\mathrm{CD}_{11 \mathrm{c}^{+} \mathrm{MHCII}}{ }^{+} \mathrm{CD} 11 \mathrm{~b}^{+}$. The subset $\mathrm{cDCs} 1$ perform a specialized function of recognizing exogenous antigens and activating the $\mathrm{CD}^{+} \mathrm{T}$ cell immune response, whereas the other subset cDCs2 is primarily responsible for presenting endogenous antigens to $\mathrm{CD}^{+} \mathrm{T}$ cells and stimulating the polarization in these cells [28-30]. CD $11 \mathrm{c}^{+} \mathrm{CD} 11 \mathrm{~b}^{+} \mathrm{cDCs}$ are the most abundant DCs in the lymphoid organs and are also found in the spleen, skin, and lung tissues. Previous studies have demonstrated that $\mathrm{CD} 11 \mathrm{c}^{+} \mathrm{CD} 11 \mathrm{~b}^{+} \mathrm{cDCs} 2$ derived $\mathrm{Th}$ ( $\mathrm{T}$ help cell) 17, Th2-immune responses in asthma, lung emphysema, and COPD [31-33]. 


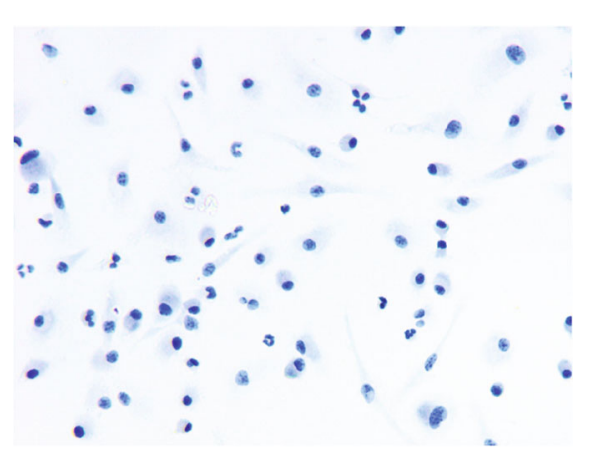

(a)
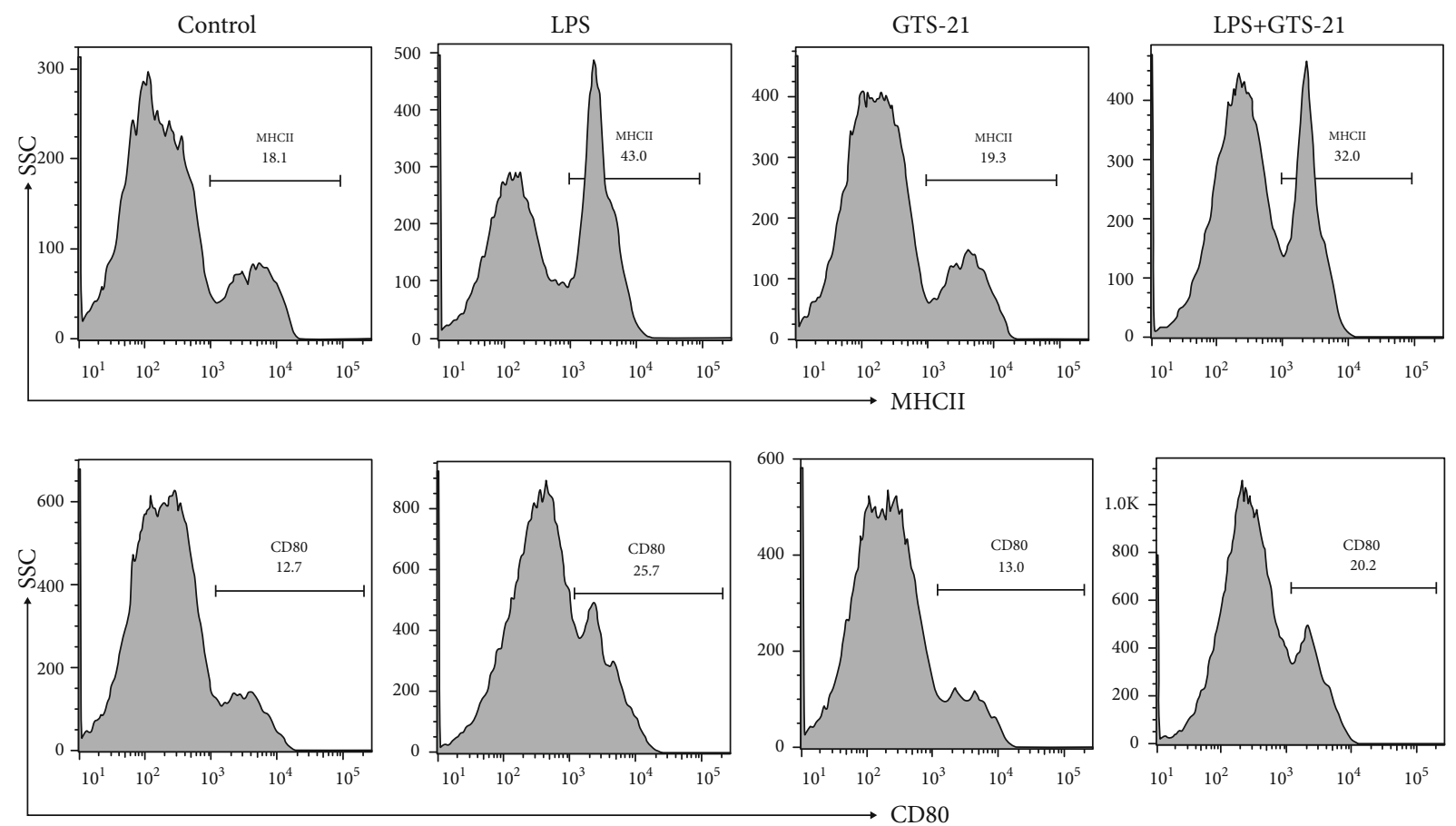

(b)

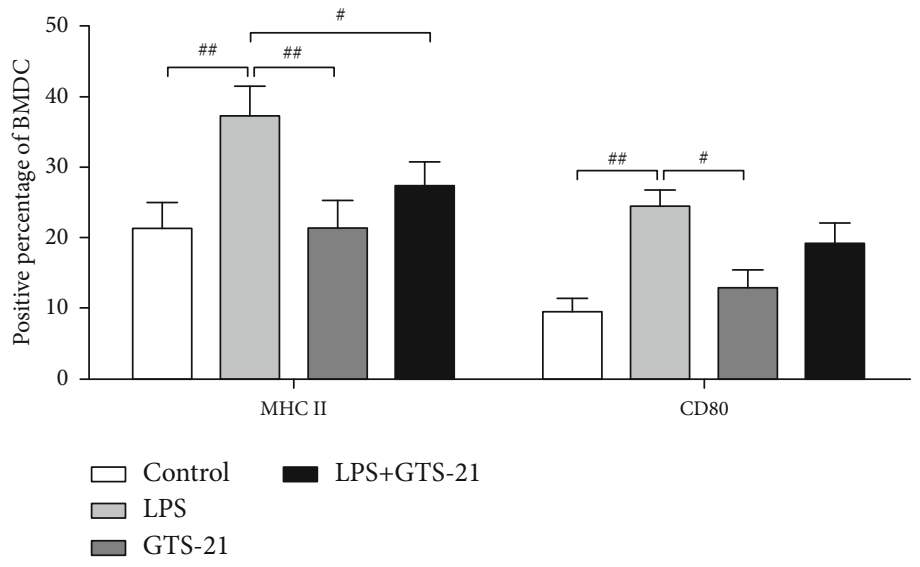

(c)

FIGURE 4: GTS-21 treatment weakened the mature differentiation of DCs in vitro. (a) The morphology of BMDCs on days 7 of culture (400x magnification). (b, c) Flow cytometry analysis was used to examine the percentages of MHCII- and CD80-positive expression on the surface of CD11 ${ }^{+}$F4/80- BMDCs. All data are shown as mean $\pm \mathrm{SD}\left(n=4-6\right.$ mice/group). ${ }^{\#} p<0.05,{ }^{\# \#} p<0.01, \#^{\# \#} p<0.001$. 


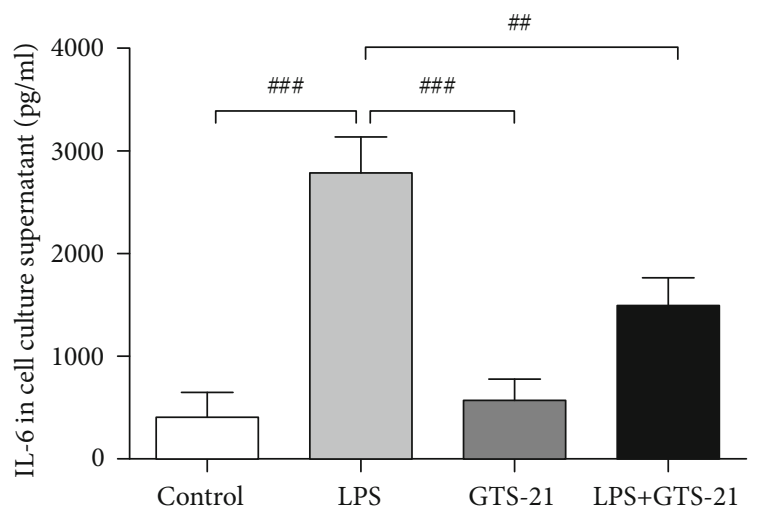

(a)

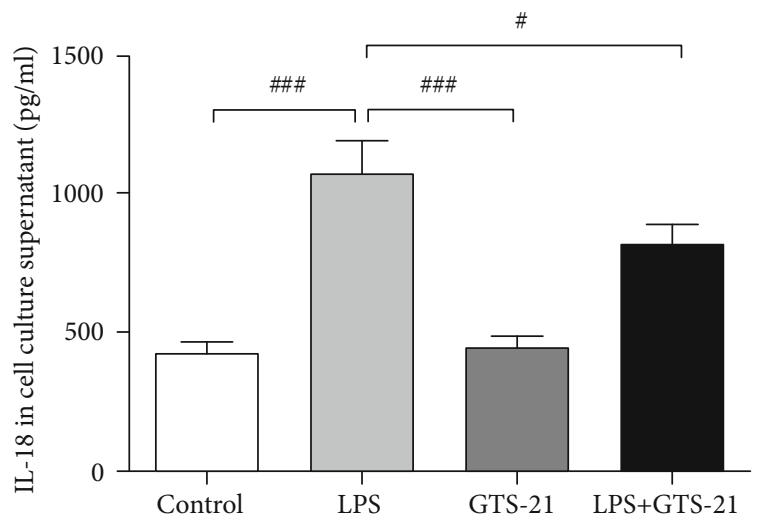

(c)

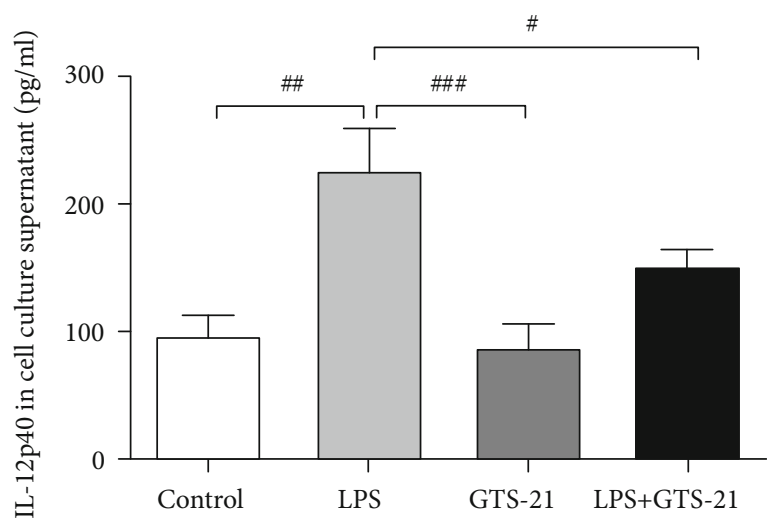

(e)

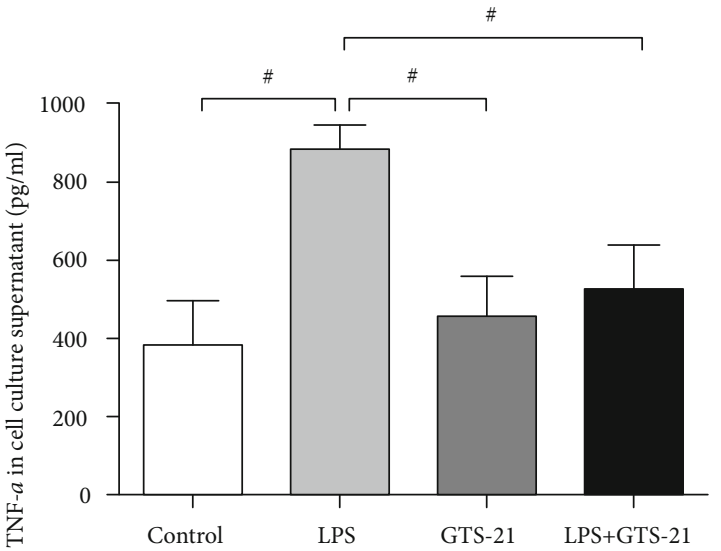

(b)

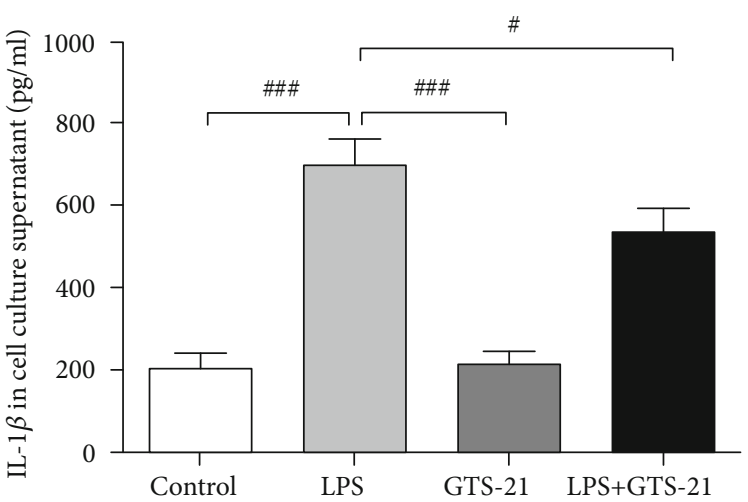

(d)

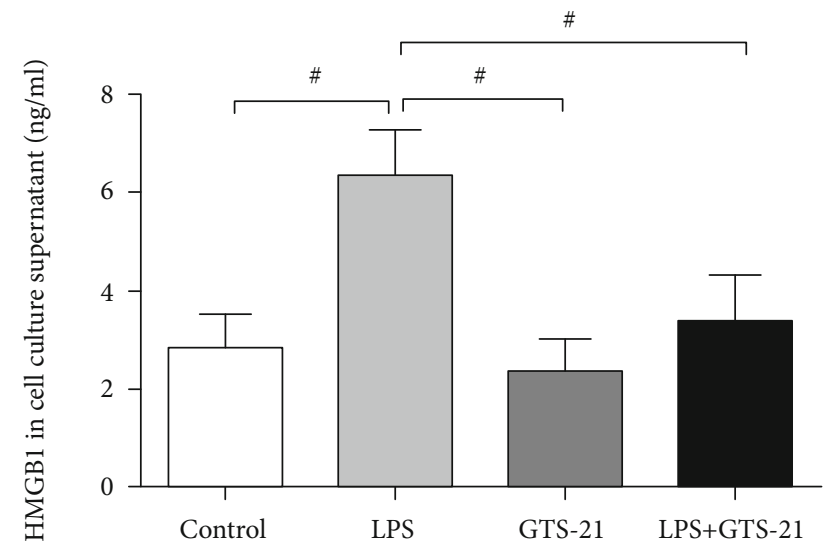

(f)

FIGURE 5: GTS-21 administration reduced DC-related proinflammatory cytokine levels in vitro. (a-d) The productions of cytokines IL-6, TNF- $\alpha$, IL-18, IL-1 $\beta$, IL-12p40, and HMGB1 in BMDC culture supernatant were measured by ELISA. All data are shown as mean \pm SD ( $n=4-6$ mice/group). ${ }^{\#} p<0.05,{ }^{\# \#} p<0.01,{ }^{\# \# \#} p<0.001$.

$\mathrm{CD}_{11} \mathrm{c}^{+} \mathrm{CD} 11 \mathrm{~b}^{+}$cDCs2 also produce pro-inflammatory cytokines such as IL-6 and IL-23 [33, 34]. Li et al. [35] reported that when $\mathrm{CD} 11 \mathrm{c}^{+} \mathrm{CD} 11 \mathrm{~b}^{+} \mathrm{cDCs} 2$ were cocultured with $\mathrm{T}$ cells or LPS, they not only displayed immunoregulatory characteristics but also played a crucial role in the induction of immune tolerance of experimental autoimmune encephalomyelitis (EAE). However, the role of $\mathrm{CD} 11 \mathrm{c}^{+} \mathrm{CD} 11 \mathrm{~b}^{+} \mathrm{cDCs} 2$ in ALI remains unclear, in particular, the relationship between cDCs 2 and CAP. In the current study, the number of CD $11 c^{+-}$
$\mathrm{CD} 11 \mathrm{~b}^{+} \mathrm{cDCs} 2$ in the spleen and lung was found be upregulated in the LPS-induced ALI mice. In addition, the results also demonstrated that GTS-21, the CAP agonist, downregulated the phenotypic expression of cDCs2 in ALI mice model. Of course, there are still some limitations in the present study. We obtained these results only with the intervention of $\alpha 7 \mathrm{nAchR}$ agonists, without the use of inhibitors or downregulation of the expression of this receptor to verify the feasibility of these mechanisms, which is what we will study next. 


\section{Conclusion}

In this study, we found that CAP could reduce the maturation of DCs and the production of DC-related proinflammatory cytokines both in vitro and in vivo and decreased the quantity of $\mathrm{cDCs}$ and $\mathrm{cDCs} 2$ in vivo. Therefore, CAP played a crucial role in the pathological process of sepsis-induced ALI through regulating the number of DCs, cDCs, and cDCs2 and also their differentiation into mature cells.

\section{Abbreviations}

$\alpha 7 \mathrm{nAChR}: \alpha 7$-subtype of the nicotinic acetylcholine receptor

ALI: $\quad$ Acute lung injury

ARDS: Acute respiratory distress syndrome

DCs: Dendritic cells

APCs: Antigen-presenting cells

cDCs: Conventional DCs

GTS-21: GTS-21 dihydrochloride

i.p.: Intraperitoneally

LPS: Lipopolysaccharide

BMDCs: Marrow-derived dendritic cells

CAP: Cholinergic anti-inflammatory pathway

cDCs: $\quad$ Conventional dendritic cells

BALF: $\quad$ Bronchoalveolar lavage fluid

RPMI: Roswell Park Memorial Institute

FBS: $\quad$ Fetal bovine serum

PBS: $\quad$ Phosphate-buffered saline

MNCs: Mononuclear cells

GM-CSF: Granulocyte-macrophage colony-stimulating factor

rmIL-4: Recombinant mouse IL-4

ELISA: Enzyme-linked immunosorbent assay

IL: Interleukin

TNF: $\quad$ Tumor necrosis factor

HMGB1: High mobility group box 1

SD: $\quad$ Standard deviation

pDCs: $\quad$ Plasmacytoid dendritic cells

cDCs1: $\quad$ Type 1 conventional dendritic cells

cDCs2: $\quad$ Type 2 conventional dendritic cells

TRALI: Transfusion-related acute lung injury.

\section{Data Availability}

Access to data is restricted.

\section{Ethical Approval}

The study was performed in compliance with the National Institutes of Health Guidelines and was approved by Huazhong University of Science and Technology (Wuhan, China) experimental animal ethics committee. The studies involving animals in the manuscript followed the recommendations in the ARRIVE guidelines [36].

\section{Conflicts of Interest}

The authors declare that they have no conflicts of interest.

\section{Authors' Contributions}

RTL, XJZ, HL, YS, and HQ designed the research; RTL, $\mathrm{XMH}, \mathrm{HBC}, \mathrm{YZ}, \mathrm{YY}, \mathrm{XHG}$, and HLG performed the experiments; RTL, HQ, and HYH analyzed the data; RTL produced the figures; RTL wrote the manuscript; HYH, XJZ, $\mathrm{HL}, \mathrm{HQ}$, and YS revised the manuscript. Haiyan Huang, Xiaojing Zou, Hong Qi, Hong Liu, and You Shang are cocorresponding authors. These authors contributed equally to this work.

\section{Acknowledgments}

A preprint has previously been published [37]. The study was supported by the National Natural Science Foundation of China (grant number 82002099).

\section{References}

[1] B. T. Thompson, R. C. Chambers, and K. D. Liu, "Acute respiratory distress syndrome," The New England Journal of Medicine, vol. 377, no. 6, pp. 562-572, 2017.

[2] J. E. Sevransky, G. S. Martin, C. Shanholtz et al., "Mortality in sepsis versus non-sepsis induced acute lung injury," Critical Care, vol. 13, no. 5, p. R150, 2009.

[3] A. Nadeem, S. F. Ahmad, N. O. Al-Harbi et al., "Inhibition of spleen tyrosine kinase signaling protects against acute lung injury through blockade of NADPH oxidase and IL-17A in neutrophils and $\gamma \delta$ T cells respectively in mice," International Immunopharmacology, vol. 68, pp. 39-47, 2019.

[4] A. Nadeem, N. O. Al-Harbi, S. F. Ahmad et al., "Blockade of interleukin-2-inducible $\mathrm{T}$-cell kinase signaling attenuates acute lung injury in mice through adjustment of pulmonary Th17/Treg immune responses and reduction of oxidative stress," International Immunopharmacology, vol. 83, p. 106369, 2020.

[5] G. Goverse, M. Stakenborg, and G. Matteoli, "The intestinal cholinergic anti-inflammatory pathway," The Journal of Physiology, vol. 594, no. 20, pp. 5771-5780, 2016.

[6] M. Yamada and M. Ichinose, "The cholinergic pathways in inflammation: a potential pharmacotherapeutic target for COPD," Frontiers in Pharmacology, vol. 3, no. 9, p. 1426, 2018.

[7] M. Yamada and M. Ichinose, "The cholinergic antiinflammatory pathway: an innovative treatment strategy for respiratory diseases and their comorbidities," Current Opinion in Pharmacology, vol. 40, pp. 18-25, 2018.

[8] Y. J. Wu, L. Wang, C. F. Ji, S. F. Gu, Q. Yin, and J. Zuo, "The role of $\alpha 7 \mathrm{nAChR}$-mediated cholinergic anti-inflammatory pathway in immune cells," Inflammation, vol. 44, no. 3, pp. 821-834, 2021.

[9] D. Liu, T. Li, H. Luo, X. Zuo, S. Liu, and S. Wu, "The effect of the cholinergic anti-inflammatory pathway on collageninduced arthritis involves the modulation of dendritic cell differentiation," Arthritis Research \& Therapy, vol. 20, no. 1, p. 263, 2018.

[10] N. O. Al-Harbi, A. Nadeem, S. F. Ahmad, M. M. Alanazi, A. A. Aldossari, and F. Alasmari, "Amelioration of sepsis-induced acute kidney injury through inhibition of inflammatory cytokines and oxidative stress in dendritic cells and neutrophils respectively in mice: role of spleen tyrosine kinase signaling," Biochimie, vol. 158, pp. 102-110, 2019. 
[11] Z. Lu, W. Chang, S. Meng et al., "Mesenchymal stem cells induce dendritic cell immune tolerance via paracrine hepatocyte growth factor to alleviate acute lung injury," Stem Cell Research \& Therapy, vol. 10, p. 372, 2019.

[12] L. Li, L. Dong, D. Zhao, F. Gao, and J. Yan, "Classical dendritic cells regulate acute lung inflammation and injury in mice with lipopolysaccharide-induced acute respiratory distress syndrome," International Journal of Molecular Medicine, vol. 44, no. 2, pp. 617-629, 2019.

[13] J. Wang, R. Li, Z. Peng et al., "GTS-21 reduces inflammation in acute lung injury by regulating $\mathrm{M} 1$ polarization and function of alveolar macrophages," Shock, vol. 51, no. 3, pp. 389-400, 2019.

[14] R. Kapur, M. Kim, R. Aslam et al., "T regulatory cells and dendritic cells protect against transfusion-related acute lung injury via IL-10," Blood, vol. 129, no. 18, pp. 2557-2569, 2017.

[15] J. W. Semple, J. Rebetz, and R. Kapur, "Transfusion-associated circulatory overload and transfusion-related acute lung injury," Blood, vol. 133, no. 17, pp. 1840-1853, 2019.

[16] L. Yang, X. Han, J. Yuan et al., "Early astragaloside IV administration attenuates experimental autoimmune encephalomyelitis in mice by suppressing the maturation and function of dendritic cells," Life Sciences, vol. 19, p. 117448, 2020.

[17] W. R. Heath and F. R. Carbone, "Dendritic cell subsets in primary and secondary T cell responses at body surfaces," Nature Immunology, vol. 10, pp. 1237-1244, 2009.

[18] C. Geurtsvan Kessel and B. Lambrecht, "Division of labor between dendritic cell subsets of the lung," Mucosal Immunology, vol. 1, pp. 442-450, 2008.

[19] A. Mildner and S. Jung, "Development and function of dendritic cell subsets," Immunity, vol. 40, no. 5, pp. 642-656, 2014.

[20] N. E. Papaioannou, N. Salei, S. Rambichler et al., "Environmental signals rather than layered ontogeny imprint the function of type 2 conventional dendritic cells in young and adult mice," Nature Communications, vol. 12, p. 464, 2021.

[21] I. Zila, D. Mokra, J. Kopincova, M. Kolomaznik, M. Javorka, and A. Calkovska, "Vagal-immune interactions involved in cholinergic anti-inflammatory pathway," Physiological Research, vol. 66, Supplement 2, pp. S139-S145, 2017.

[22] I. Vieira-Alves, L. M. C. Coimbra-Campos, M. Sancho, R. F. da Silva, S. F. Cortes, and V. S. Lemos, "Role of the $\alpha 7$ nicotinic acetylcholine receptor in the pathophysiology of atherosclerosis," Frontiers in Physiology, vol. 11, article 621769, 2020.

[23] S. Li, D. Qi, J. N. Li, X. Y. Deng, and D. X. Wang, "Vagus nerve stimulation enhances the cholinergic anti-inflammatory pathway to reduce lung injury in acute respiratory distress syndrome via STAT3," Cell Death Discovery, vol. 7, no. 1, p. 63, 2021.

[24] Y. Deng, S. L. Guo, B. Wei, X. C. Gao, Y. C. Zhou, and J. Q. Li, "Activation of nicotinic acetylcholine $\alpha 7$ receptor attenuates progression of monocrotaline-induced pulmonary hypertension in rats by downregulating the NLRP3 inflammasome," Frontiers in Pharmacology, vol. 10, p. 128, 2019.

[25] A. Gardner and B. Ruffell, "Dendritic cells and cancer immunity," Trends in Immunology, vol. 37, no. 12, pp. 855-865, 2016.

[26] J. Liu, P. Zhang, Q. Yu et al., "Losartan inhibits conventional dendritic cell maturation and Th1 and Th17 polarization responses: novel mechanisms of preventive effects on lipopolysaccharide-induced acute lung injury," International Journal of Molecular Medicine, vol. 29, pp. 269-276, 2012.
[27] J. Liu, P. S. Zhang, Q. Yu, L. Liu, Y. Yang, and H. B. Qiu, "Kinetic and distinct distribution of conventional dendritic cells in the early phase of lipopolysaccharide-induced acute lung injury," Molecular Biology Reports, vol. 39, no. 12, pp. 10421-10431, 2012.

[28] B. Janela, A. A. Patel, M. C. Lau et al., "A subset of type I conventional dendritic cells controls cutaneous bacterial infections through VEGF $\alpha$-mediated recruitment of neutrophils," Immunity, vol. 50, no. 4, pp. 1069-1083.e8, 2019.

[29] A. Gardner, P. Á. de Mingo, and B. Ruffell, "Dendritic cells and their role in immunotherapy," Frontiers in Immunology, vol. 11, p. 924, 2020.

[30] X. Zhang, M. Artola-Boran, A. Fallegger et al., "IRF4 expression is required for the immunoregulatory activity of conventional type 2 dendritic cells in settings of chronic bacterial infection and cancer," Journal of Immunology, vol. 205, no. 7, pp. 1933-1943, 2020.

[31] T. Naessens, Y. Morias, E. Hamrud et al., "Human lung conventional dendritic cells orchestrate lymphoid neogenesis during chronic obstructive pulmonary disease," American Journal of Respiratory and Critical Care Medicine, vol. 202, no. 4, pp. 535-548, 2020.

[32] S. R. Beaty, C. E. Rose Jr., and S. S. Sung, "Diverse and potent chemokine production by lung CD11bhigh dendritic cells in homeostasis and in allergic lung inflammation," Journal of Immunology, vol. 178, no. 3, pp. 1882-1895, 2007.

[33] M. Plantinga, M. Guilliams, M. Vanheerswynghels et al., "Conventional and monocyte-derived CD11b(+) dendritic cells initiate and maintain T helper 2 cell-mediated immunity to house dust mite allergen," Immunity, vol. 38, no. 2, pp. 322335, 2013.

[34] A. Schlitzer, N. McGovern, P. Teo et al., "IRF4 transcription factor-dependent $\mathrm{CD} 11 \mathrm{~b}+$ dendritic cells in human and mouse control mucosal IL-17 cytokine responses," Immunity, vol. 38, no. 5, pp. 970-983, 2013.

[35] H. Li, G. X. Zhang, Y. Chen et al., "CD11c+CD11b+ dendritic cells play an important role in intravenous tolerance and the suppression of experimental autoimmune encephalomyelitis," Journal of Immunology, vol. 181, no. 4, pp. 2483-2493, 2008.

[36] C. Kilkenny, W. J. Browne, I. C. Cuthill, M. Emerson, and D. G. Altman, "Improving bioscience research reporting: the ARRIVE guidelines for reporting animal research," PLoS Biology, vol. 8, no. 6, p. e1000412, 2010.

[37] R. Li, X. Hu, H. Chen et al., "Role of cholinergic antiinflammatory pathway in protecting sepsis-induced acute lung injury through regulation of the dendritic cells. 